\title{
MIMO 雷达最大似然参数估计
}

\author{
夏威*，何子述，廖羽宇 \\ 电子科技大学, 电子工程学院, 成都 611731 \\ * 通信作者. E-mail: wx@uestc.edu.cn
}

收稿日期: 2009-11-17; 接受日期: 2010-03-19

国家自然科学基金 (批准号: 60672044) 资助项目

\begin{abstract}
摘要 多输入多输出 (MIMO) 雷达使用多个天线同时发射多个独立探测信号, 并使用多个天线接收 目标回波信号. 本文考虑了发射空域分集、相干接收 MIMO 雷达模型及其最大似然 (ML) 参数估计 方法. 基于最大似然准则, 本文推导了两种渐近最大似然算法. 仿真实验的结果表明, 在均匀噪声模 型中, 其中一种渐近算法与基于延迟求和波束形成的最大似然算法性能接近, 而另一种渐近算法性 能略差, 但具有较低的计算复杂度. 而在非均匀噪声模型中, 本文所提出的两种渐近最大似然算法的 性能均优于基于延迟求和波束形成的最大似然算法.
\end{abstract}

关键词 多输入多输出 (MIMO) 雷达 参数估计 最大似然估计

\section{1 引言}

多输入多输出 (multiple-input multiple-output, MIMO) 雷达对目标雷达横截面积 (radar cross section, RCS) 起伏不敏感 ${ }^{[1-3]}$, 具有高分辨的空间谱估计能力 ${ }^{[4-6]}$ 和参数估计性能 ${ }^{[7]}$, 可以实现灵活 的发射波束方向图设计 ${ }^{[8-10]}$ 和功率分配 ${ }^{[11]}$, MIMO 雷达的概念和有关的信号处理技术正受到越来 越多的关注 ${ }^{[12-21]}$.

现有的 MIMO 雷达模型通常可以分为两类 $[3,6,12,13,21]$. 第一类 MIMO 雷达模型采用较小阵元间 距的阵列作为发射和接收阵列, 可实现相干 (coherent) 发射和接收 [4,5,7-10,12,14,21]. 第二类 MIMO 雷 达模型的发射阵列的阵元间距较大, 可在发射端实现空域分集 (spatial diversity). 如果接收阵列的阵 元间距较大, 可实现接收端的空域分集 $[1,3,11,18,19,21]$, 在这种情况下, MIMO 雷达可实现目标的非相干 检测, 但不能从接收的非相干目标回波中提取目标位置和速度等信息. 在第二类模型中, 如果接收阵列 的阵元间距较小, 则在接收端可实现相干处理, 如实现有源测向 (active direction finding) 功能等 $[2,21]$. 如果发射和接收阵列由非相干的子阵构成, 则可同时获得空域分集增益和相干处理增益 ${ }^{[6,20,21]}$.

本文将考虑发射端空域分集、接收端相干的 MIMO 雷达模型 ${ }^{[2]}$ 及其最大似然 (maximum likelihood, ML) 参数估计问题. 在 MIMO 雷达模型推导过程中, 文献 [2] 将目标的独立散射体建模为与发 射和接收阵列平行的均匀线阵 (uniform linear array, ULA) ${ }^{1)}$. 尽管没有明确指出, 但文献 [2] 的结论表 明, 在上述假设下获得的 MIMO 雷达模型与目标散射体的几何分布并无直接联系. 此外, 文献 [2] 假

1) 文献 [2] 同时也假设发射和接收阵列为均匀线阵 
设目标相对于接收阵列不存在角度扩展, 于是, 接收阵列接收到的目标回波信号是完全相干的. 借鉴 无线通信系统的物理散射建模方法, 本文将推导在不同情况下发射端空域分集, 接收端相干的 MIMO 雷达模型. 本文的推导结果表明, 当接收阵列所观测的目标不存在角度扩展时, 可以获得与文献 $[2]$ 相同的模型. 文献 [21] 包含了 MIMO 雷达信号模型的更为详细的论述, 并深入讨论了其他参数估计 算法.

文献 [2] 使用最大似然方法解决了该 MIMO 雷达模型的有源测向问题. 在文献 [2] 中, 假设雷达 系统的噪声是加性均匀白噪声 (uniform noise), 即在各接收阵元处的噪声功率相等. 此外, 文献 [2] 只 给出了目标角度的估计, 而没有给出与目标的衰落向量的估计. 除此之外, 按文献 [2] 的方法直接求解 的计算量较大. 基于最大似然估计的基本原理, 本文推导了两种渐近最大似然算法. 其中一种渐近算 法具有良好的估计性能, 但运算量较大; 而另一种渐近算法性能略差, 但具有较低的运算量. 本文同时 给出了目标衰落向量的渐近最大似然估计. 需要指出的是, 本文所得到的算法受到了阵列信号处理中 的解耦最大似然 (decoupled ML, DEML) 算法 ${ }^{[22]}$ 的启发. 然而, 由于本文所考虑信号模型与阵列信 号处理的模型不同, 因此 DEML 的结论不能直接应用于 MIMO 雷达模型, 而最终得到的结论也是不 同的.

\section{2 信号模型}

考虑图 1 和 2 所示由 $K$ 个发射阵元和 $M$ 个接收阵元构成的 MIMO 雷达系统. 考虑分布式目标 模型, 即假设目标由多个独立的小散射体组成. 假设发射阵列的阵元间距足够大, 使得前向信道 ${ }^{2)}$ 满 足正交条件 ${ }^{[1,3]}$. 在这种情况下, 独立的探测信号通过不相干的路径照射到目标, 因此将经历相互独立 的衰落, 从而实现发射端的空域分集. 注意到, 这里并未假设发射阵列的布阵情况, 发射阵列只需满足 文献 $[1,3]$ 所给出的空域分集的充分条件. 在雷达接收阵列, 目标入射角的角度扩展与目标和接收阵列 的相对位置有关. 为了简化讨论, 本文假设各天线阵元之间时间同步和相位同步.

考虑如图 1 所示的情况. 假设从第 $k$ 个发射阵元发出的窄带探测信号 $s_{k}(t)$, 经目标反射后, 接收 阵列接收到来自 $L$ 个可分辨的目标散射体的反向散射信号, 于是 (无噪声的) 连续时间单输入多输出 (single-input multiple-output, SIMO) 基带信号模型可以表示为

$$
\boldsymbol{x}_{k}(t)=\sum_{l=1}^{L} \boldsymbol{a}\left(\theta_{l}\right) \tilde{\alpha}_{k, l} s_{k}(t-\tau)=\boldsymbol{h}_{k} s_{k}(t-\tau), \quad k=1, \ldots, K,
$$

其中, $\boldsymbol{a}(\theta)$ 是接收阵列的响应向量, $\theta_{l}$ 和 $\tilde{\alpha}_{k, l}$ 分别是第 $l$ 个散射体反向散射回波的入射角和复值路径 增益, $\tau$ 是目标的路径延迟. 不失一般性, 假设 $\tau=0$. 注意到在窄带假设下, 不同散射体之间的时延差 异可忽略不计. 假设接收阵列是均匀线阵, 即

$$
\boldsymbol{a}(\theta)=\left[\begin{array}{llll}
1 & e^{-j 2 \pi \sin (\theta) d_{r}} & \ldots & e^{-j 2 \pi \sin (\theta)(M-1) d_{r}}
\end{array}\right]^{\mathrm{T}} \in \mathbb{C}^{M \times 1},
$$

其中, $d_{r}$ 是以载波波长为单位的阵元间距, 上标 $(\cdot)^{\mathrm{T}}$ 表示矩阵或向量的转置运算, $\mathbb{C}$ 表示复数域. 可 以证明 [1], 如果目标由大量随机均匀分布的各向同性的独立散射体所构成, 那么当发射阵列的阵元间 距足够大时, $\tilde{\alpha}_{k, l}(k=1, \ldots, K, l=1, \ldots, L)$ 是零均值、单位方差的, 独立同分布的 (i.i.d.) 圆对称复 高斯随机变量 (circularly symmetric complex Gaussian random variables) ${ }^{3)}$.

2) 前向信道指从发射阵列到目标的信号传播路径

3) 圆对称复高斯随机变量 $z=x+j y \sim \mathcal{C N}\left(0, \sigma^{2}\right)$, 实部 $x$ 和虚部 $y$ 是 i.i.d. 的, $x, y \sim \mathcal{N}\left(0, \sigma^{2} / 2\right)$ 


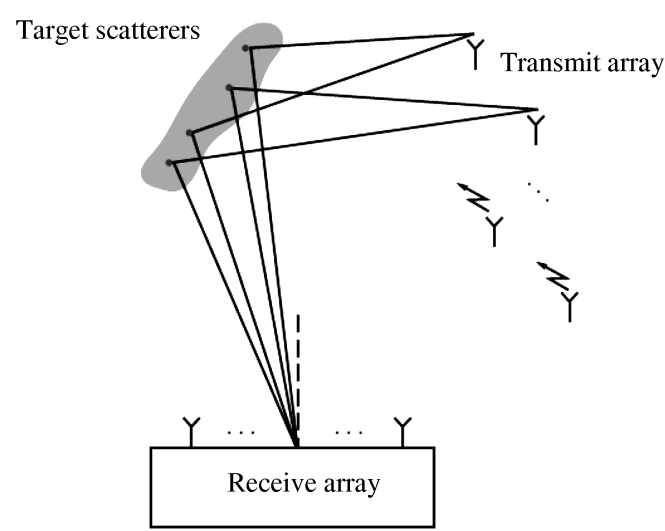

图 1 目标存在角度扩展

Figure 1 Target with angle spread

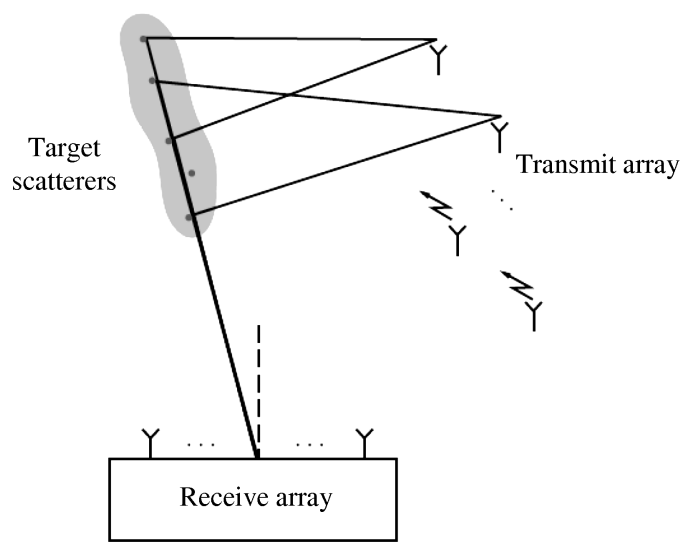

图 2 目标不存在角度扩展

Figure 2 Target without angle spread

由式 (1) 知, 第 $k$ 个 SIMO 信道向量可以表示为

$$
\boldsymbol{h}_{k}=\sum_{l=1}^{L} \boldsymbol{a}\left(\theta_{l}\right) \tilde{\alpha}_{k, l}=\boldsymbol{A}(\boldsymbol{\theta}) \tilde{\boldsymbol{\alpha}}_{k}, \quad k=1, \ldots, K,
$$

其中,

$$
\begin{aligned}
& \boldsymbol{\theta}=\left[\begin{array}{llll}
\theta_{1} & \theta_{2} & \ldots & \theta_{L}
\end{array}\right]^{\mathrm{T}} \in \mathbb{C}^{L \times 1}, \\
& \tilde{\boldsymbol{\alpha}}_{k}=\left[\begin{array}{llll}
\tilde{\alpha}_{k, 1} & \tilde{\alpha}_{k, 2} & \ldots & \tilde{\alpha}_{k, L}
\end{array}\right]^{\mathrm{T}} \in \mathbb{C}^{L \times 1}, \\
& \boldsymbol{A}(\boldsymbol{\theta})=\left[\begin{array}{llll}
\boldsymbol{a}\left(\theta_{1}\right) & \boldsymbol{a}\left(\theta_{2}\right) & \ldots & \boldsymbol{a}\left(\theta_{L}\right)
\end{array}\right] \in \mathbb{C}^{M \times L} .
\end{aligned}
$$

考虑 $K$ 个独立发射信号, 于是, 离散时间 MIMO 雷达的基带接收信号可以表示为

$$
\boldsymbol{x}(n)=\sum_{k=1}^{K} \boldsymbol{h}_{k} s_{k}(n)+\boldsymbol{v}(n)=\boldsymbol{A}(\boldsymbol{\theta}) \mathcal{A} \boldsymbol{s}(n)+\boldsymbol{v}(n),
$$

其中, $\mathcal{A}=\left[\begin{array}{cccc}\tilde{\boldsymbol{\alpha}}_{1} & \tilde{\boldsymbol{\alpha}}_{2} & \cdots & \tilde{\boldsymbol{\alpha}}_{K}\end{array}\right] \in \mathbb{C}^{L \times K}$ 是目标的衰落矩阵, $\boldsymbol{x}[n]$ 和 $\boldsymbol{s}[n]$ 分别是基带的接收信号向 量和发射信号向量:

$$
\begin{aligned}
& \boldsymbol{x}[n]=\left[\begin{array}{llll}
x_{1}[n] & x_{2}[n] & \ldots & x_{M}[n]
\end{array}\right]^{\mathrm{T}} \in \mathbb{C}^{M \times 1}, \\
& \boldsymbol{s}[n]=\left[\begin{array}{llll}
s_{1}[n] & s_{2}[n] & \ldots & s_{K}[n]
\end{array}\right]^{\mathrm{T}} \in \mathbb{C}^{K \times 1},
\end{aligned}
$$

噪声向量 $\boldsymbol{v}[n]$ 与 $\boldsymbol{x}[n]$ 的定义类似.

现在考虑如图 2 所示 MIMO 雷达信号模型. 在这种情况下, 假设接收阵列无法分辨来自同一目 标的不同散射体的反向散射信号, 于是

$$
\boldsymbol{x}_{k}(t)=\sum_{l=1}^{L} \boldsymbol{a}(\theta) \tilde{\alpha}_{k, l} s_{k}(t-\tau)=\boldsymbol{a}(\theta) \sum_{l=1}^{L} \tilde{\alpha}_{k, l} s_{k}(t-\tau) \triangleq \boldsymbol{a}(\theta) \alpha_{k} s_{k}(t-\tau), \quad k=1, \ldots, K,
$$


其中, $\theta$ 是目标反射信号的到达角, $\alpha_{k}=\sum_{l=1}^{L} \tilde{\alpha}_{k, l}$ 是零均值、单位方差的圆对称复高斯随机变量. 假 设 $\tau=0$. 与式 (4) 类似, 离散时间 MIMO 雷达基带接收信号可以表示为

$$
\boldsymbol{x}[n]=\sum_{k=1}^{K} \boldsymbol{h}_{k} s_{k}[n]+\boldsymbol{v}[n]=\boldsymbol{H} \boldsymbol{s}[n]+\boldsymbol{v}[n],
$$

其中,

$$
\boldsymbol{H}=\boldsymbol{a}(\theta) \boldsymbol{\alpha}^{\mathrm{T}}
$$

是 MIMO 雷达信道矩阵,

$$
\boldsymbol{\alpha}=\left[\begin{array}{cccc}
\tilde{\alpha}_{1} & \tilde{\alpha}_{2} & \ldots & \tilde{\alpha}_{K}
\end{array}\right]^{\mathrm{T}} \in \mathbb{C}^{K \times 1}
$$

是目标的衰落向量. 注意到, 对于远场目标, 当目标的尺寸远小于目标与接收阵列之间的距离时 ${ }^{[2]}$, 如 果目标的散射体到达角的差异超过了接收阵列的角度分辨能力, 则接收阵列所观测到的目标回波信号 也由式 (6) 描述.

式 (4) 和 (6) 分别给出了接收阵列所观测的目标具有角度扩展和没有角度扩展情况下 MIMO 雷 达的信号模型, 式 (6) 可以看作是式 (4) 的极端情况. 限于篇幅, 本文仅考虑 MIMO 雷达模型 (6) 的最 大似然参数估计问题. 本文考虑准静态衰落 (quasi-static fading) 情况, 即假设在最大似然估计的观测 时间间隔内衰落向量 $\boldsymbol{\alpha}$ 是常值; 而在不同观测时间间隔内, $\boldsymbol{\alpha}$ 是随机变化的. 假设发射信号向量 $\boldsymbol{s}[n]$ 的各分量是零均值的, 并相互正交, 且协方差矩阵为 $\boldsymbol{R}_{\boldsymbol{s}}=E\left\{\boldsymbol{s}[n] \boldsymbol{s}^{\mathrm{H}}[n]\right\}=\sigma_{s}^{2} \boldsymbol{I}_{K}$, 其中, $\sigma_{s}^{2}$ 是发射信 号的平均功率, $\boldsymbol{I}_{K}$ 是 $K$ 维单位矩阵, $\mathrm{E}\{\cdot\}$ 和上标 $(\cdot)^{\mathrm{H}}$ 分别表示数学期望和矩阵或向量的转置共轭运 算. 假设噪声向量 $\boldsymbol{v}[n]$ 是零均值, 时间白的 (temporally white) 圆对称复高斯随机过程, 且协方差矩阵 为 $\boldsymbol{R}_{\boldsymbol{v} \boldsymbol{v}}=\mathrm{E}\left\{\boldsymbol{v}[n] \boldsymbol{v}^{\mathrm{H}}[\nu]\right\}=\boldsymbol{Q} \delta_{n, \nu}$, 其中, $\delta_{n, \nu}$ 是 Kronecker delta 函数. 假设矩阵 $\boldsymbol{Q} \in \mathbb{C}^{M \times M}$ 是未知的. 假设噪声 $\boldsymbol{v}[n]$ 与发射信号 $\boldsymbol{s}[n]$ 不相关.

\section{3 最大似然估计}

\section{1 延迟求和波束形成}

文献 [2] 使用延迟求和波束形成器 (delay-and-sum beamformer) 估计目标角度

$$
\hat{\theta}=\arg \max _{\theta} \sum_{n=0}^{N-1}\left\|\boldsymbol{a}^{\mathrm{H}}(\theta) \boldsymbol{x}[n]\right\|^{2},
$$

其中, $\|\cdot\|$ 表示复数的模, $\{\boldsymbol{x}[n]\}_{n=0}^{N-1}$ 是观测到的接收信号向量, $N$ 是观测样本数. 注意到在单目标情 况下, 延迟求和波束形成器是角度 $\theta$ 的最大似然估计. 利用接收信号的样本协方差矩阵

$$
\hat{\boldsymbol{R}}_{\boldsymbol{x} \boldsymbol{x}}=\frac{1}{N} \sum_{n=0}^{N-1} \boldsymbol{x}[n] \boldsymbol{x}^{\mathrm{H}}[n],
$$

式 (9) 可改写为

$$
\hat{\theta}=\arg \max _{\theta} \boldsymbol{a}^{\mathrm{H}}(\theta) \hat{\boldsymbol{R}}_{\boldsymbol{x} \boldsymbol{x}} \boldsymbol{a}(\theta) .
$$

文献 [2] 并未给出目标衰落向量 $\boldsymbol{\alpha}$ 的估计. 


\section{2 角度和衰落向量的渐近最大似然估计}

本小节将从最大似然的基本原理 ${ }^{[21-24]}$ 出发, 推导目标角度和衰落向量的渐近最大似然估计. 理 论结果表明, 由于信号传输的特点, MIMO 雷达系统对 RCS 起伏不敏感.

由于噪声向量 $\boldsymbol{v}[n]$ 是高斯的, 考虑接收信号 $\{\boldsymbol{x}[n]\}_{n=0}^{N-1}$ 的负对数似然函数 (忽略常数项)

$$
J=\ln |\boldsymbol{Q}|+\operatorname{tr}\left\{\boldsymbol{Q}^{-1} \frac{1}{N} \sum_{n=0}^{N-1}(\boldsymbol{x}[n]-\boldsymbol{H} \boldsymbol{s}[n])(\boldsymbol{x}[n]-\boldsymbol{H} \boldsymbol{s}[n])^{\mathrm{H}}\right\},
$$

其中, $|\cdot|$ 和 $\operatorname{tr}\{\cdot\}$ 分别表示矩阵的行列式和迹 (trace). 可以证明, 噪声协方差矩阵 $\boldsymbol{Q}$ 的最大似然估计 为 $[21-23]$

$$
\hat{\boldsymbol{Q}}=\frac{1}{N} \sum_{n=0}^{N-1}(\boldsymbol{x}[n]-\hat{\boldsymbol{H}} \boldsymbol{s}[n])(\boldsymbol{x}[n]-\hat{\boldsymbol{H}} \boldsymbol{s}[n])^{\mathrm{H}},
$$

其中, 信道矩阵的估计 $\hat{\boldsymbol{H}}$ 使样本协方差矩阵

$$
\boldsymbol{G}=\frac{1}{N} \sum_{n=0}^{N-1}(\boldsymbol{x}[n]-\boldsymbol{H} \boldsymbol{s}[n])(\boldsymbol{x}[n]-\boldsymbol{H} \boldsymbol{s}[n])^{\mathrm{H}}
$$

的行列式 $J_{G} \triangleq|\boldsymbol{G}|$ 取得极小值. 与 $\hat{\boldsymbol{R}}_{\boldsymbol{x} \boldsymbol{x}}$ 类似, 定义发射信号 $\{\boldsymbol{s}[n]\}_{n=0}^{N-1}$ 样本协方差矩阵 $\hat{\boldsymbol{R}}_{\boldsymbol{s} \boldsymbol{s}}$, 并定义 发射信号和接收信号的样本互协方差矩阵

$$
\hat{\boldsymbol{R}}_{\boldsymbol{x} \boldsymbol{s}}=\frac{1}{N} \sum_{n=0}^{N-1} \boldsymbol{x}[n] \boldsymbol{s}^{\mathrm{H}}[n] .
$$

于是, 式 (14) 可以改写为 ${ }^{4)}$

$$
\begin{aligned}
\boldsymbol{G} & =\hat{\boldsymbol{R}}_{\boldsymbol{x} \boldsymbol{x}}-\hat{\boldsymbol{R}}_{\boldsymbol{x} s} \boldsymbol{H}^{\mathrm{H}}-\boldsymbol{H} \hat{\boldsymbol{R}}_{\boldsymbol{x} \boldsymbol{s}}^{\mathrm{H}}+\boldsymbol{H} \hat{\boldsymbol{R}}_{\boldsymbol{s}} \boldsymbol{H}^{\mathrm{H}} \\
& =\hat{\boldsymbol{R}}_{\boldsymbol{x} \boldsymbol{x}}-\hat{\boldsymbol{R}}_{\boldsymbol{x} \boldsymbol{s}} \hat{\boldsymbol{R}}_{\boldsymbol{s} \boldsymbol{s}}^{-1} \hat{\boldsymbol{R}}_{\boldsymbol{x} \boldsymbol{s}}^{\mathrm{H}}+\left(\boldsymbol{H}-\hat{\boldsymbol{R}}_{\boldsymbol{x} \boldsymbol{s}} \hat{\boldsymbol{R}}_{\boldsymbol{s}}^{-1}\right) \hat{\boldsymbol{R}}_{\boldsymbol{s} \boldsymbol{s}}\left(\boldsymbol{H}^{\mathrm{H}}-\hat{\boldsymbol{R}}_{\boldsymbol{s} \boldsymbol{s}}^{-1} \hat{\boldsymbol{R}}_{\boldsymbol{x} \boldsymbol{s}}^{\mathrm{H}}\right) \geqslant \hat{\boldsymbol{R}}_{\boldsymbol{x} \boldsymbol{x}}-\hat{\boldsymbol{R}}_{\boldsymbol{x} \boldsymbol{s}} \hat{\boldsymbol{R}}_{\boldsymbol{s} \boldsymbol{s}}^{-1} \hat{\boldsymbol{R}}_{\boldsymbol{x} \boldsymbol{s}}^{\mathrm{H}},
\end{aligned}
$$

当且仅当

$$
\hat{\boldsymbol{H}}=\hat{\boldsymbol{R}}_{\boldsymbol{x} \boldsymbol{s}} \hat{\boldsymbol{R}}_{\boldsymbol{s} \boldsymbol{s}}^{-1}
$$

时, 式 (16) 取等号. 因此, $\hat{\boldsymbol{H}}$ 将极小化样本协方差矩阵 $\boldsymbol{G}$ 的特征值 [23]. 由于行列式是特征值的单调 非递减函数, 于是, $\hat{\boldsymbol{H}}$ 将极小化行列式函数 $J_{G}$. 将式 (17) 代入式 (13), 可以得到噪声的样本协方差矩 阵 $\boldsymbol{Q}$ 的估计

$$
\hat{\boldsymbol{Q}}=\hat{\boldsymbol{R}}_{\boldsymbol{x} \boldsymbol{x}}-\hat{\boldsymbol{R}}_{\boldsymbol{x} s} \hat{\boldsymbol{R}}_{s s}^{-1} \hat{\boldsymbol{R}}_{\boldsymbol{x} s}^{\mathrm{H}} .
$$

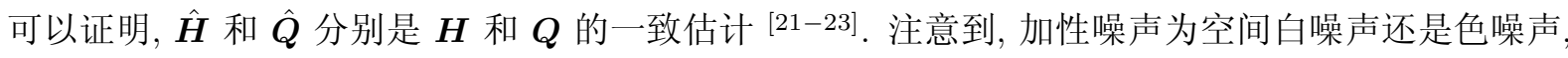
并不影响信道矩阵 $\boldsymbol{H}$ 的最大似然估计.

将式 (17) 和 (18) 代入式 (16), 可以将行列式函数 $J_{G}=|\boldsymbol{G}|$ 改写为

$$
\begin{aligned}
J_{G} & =\left|\hat{\boldsymbol{Q}}+(\boldsymbol{H}-\hat{\boldsymbol{H}}) \hat{\boldsymbol{R}}_{s s}(\boldsymbol{H}-\hat{\boldsymbol{H}})^{\mathrm{H}}\right|=|\hat{\boldsymbol{Q}}|\left|\boldsymbol{I}+\hat{\boldsymbol{Q}}^{-1}(\boldsymbol{H}-\hat{\boldsymbol{H}}) \hat{\boldsymbol{R}}_{s s}(\boldsymbol{H}-\hat{\boldsymbol{H}})^{\mathrm{H}}\right| \\
& =|\hat{\boldsymbol{Q}}|\left|\boldsymbol{I}+\hat{\boldsymbol{R}}_{s s}(\boldsymbol{H}-\hat{\boldsymbol{H}})^{\mathrm{H}} \hat{\boldsymbol{Q}}^{-1}(\boldsymbol{H}-\hat{\boldsymbol{H}})\right| .
\end{aligned}
$$

4) 矩阵不等式 $\boldsymbol{A} \geqslant \boldsymbol{B}$ 表示矩阵 $\boldsymbol{A}-\boldsymbol{B}$ 是半正定 (positive semi-definite) 矩阵 
这里假设 $\hat{\boldsymbol{Q}}$ 是非奇异的. 如果 $\hat{\boldsymbol{Q}}$ 是奇异的, 可以使用对角加载 (diagonal loading) 技术, 即, 使用 $\hat{\boldsymbol{Q}}+\epsilon \boldsymbol{I}_{M}$ 代替 $\hat{\boldsymbol{Q}}$, 其中, $\epsilon>0$ 是对角加载因子. 此外, 在上面的推导中利用了矩阵等式 $|\boldsymbol{I}+\boldsymbol{A} \boldsymbol{B}|=$ $|\boldsymbol{I}+\boldsymbol{B} \boldsymbol{A}|$, 其中, $\boldsymbol{I}$ 是单位矩阵, $\boldsymbol{A}$ 和 $\boldsymbol{B}$ 是矩阵 [24]. 采用文献 [22] 所用的方法, 对于大样本数 $N$, 极 小化式 (19) 渐近等价于下面的极小化问题:

$$
\min _{\boldsymbol{H}} \operatorname{tr}\left\{\hat{\boldsymbol{R}}_{\boldsymbol{s} \boldsymbol{s}}(\boldsymbol{H}-\hat{\boldsymbol{H}})^{\mathrm{H}} \hat{\boldsymbol{Q}}^{-1}(\boldsymbol{H}-\hat{\boldsymbol{H}})\right\} .
$$

当 $N$ 充分大时, 有 $\hat{\boldsymbol{R}}_{s} \approx \sigma_{s}^{2} \boldsymbol{I}_{K}$. 因此, 式 (20) 的极小化问题可进一步分解为 $K$ 个极小化问题,

$$
\min _{\boldsymbol{h}_{k}}\left(\hat{\boldsymbol{h}}_{k}-\boldsymbol{h}_{k}\right)^{\mathrm{H}} \hat{\boldsymbol{Q}}^{-1}\left(\hat{\boldsymbol{h}}_{k}-\boldsymbol{h}_{k}\right), \quad k=1, \ldots, K,
$$

其中, $\hat{\boldsymbol{h}}_{k}$ 是矩阵 $\hat{\boldsymbol{H}}$ 的第 $k$ 列向量. 由式 (7) 有, $\hat{\boldsymbol{h}}_{k}=\hat{\alpha}_{k} \boldsymbol{a}(\hat{\theta})$, 其中, $\hat{\alpha}_{k}$ 和 $\hat{\theta}$ 分别是目标衰落系数和 角度的估计. 于是,

$$
\hat{\alpha}_{k}=\frac{\boldsymbol{a}^{\mathrm{H}}(\hat{\theta}) \hat{\boldsymbol{Q}}^{-1} \hat{\boldsymbol{h}}_{k}}{\boldsymbol{a}^{\mathrm{H}}(\hat{\theta}) \hat{\boldsymbol{Q}}^{-1} \boldsymbol{a}(\hat{\theta})}, \quad k=1, \ldots, K .
$$

因此, 衰落向量 $\boldsymbol{\alpha}$ 的估计可以表示为

$$
\hat{\boldsymbol{\alpha}}^{\mathrm{T}}=\frac{\boldsymbol{a}^{\mathrm{H}}(\hat{\theta}) \hat{\boldsymbol{Q}}^{-1} \hat{\boldsymbol{H}}}{\boldsymbol{a}^{\mathrm{H}}(\hat{\theta}) \hat{\boldsymbol{Q}}^{-1} \boldsymbol{a}(\hat{\theta})} .
$$

将式 (22) 代入式 (21), 可以得到使得式 (21) 取得极小值的目标角度 $\theta$ 的估计

$$
\hat{\theta}=\arg \max _{\theta} \frac{\left\|\boldsymbol{a}^{\mathrm{H}}(\theta) \hat{\boldsymbol{Q}}^{-1} \hat{\boldsymbol{h}}_{k}\right\|^{2}}{\boldsymbol{a}^{\mathrm{H}}(\theta) \hat{\boldsymbol{Q}}^{-1} \boldsymbol{a}(\theta)}=\arg \max _{\theta} \hat{\boldsymbol{h}}_{k}^{\mathrm{H}} \hat{\boldsymbol{Q}}^{-\frac{1}{2}} \boldsymbol{\Pi} \hat{\boldsymbol{Q}}^{-\frac{\mathrm{H}}{2}} \hat{\boldsymbol{h}}_{k},
$$

其中, $\boldsymbol{\Pi} \triangleq \tilde{\boldsymbol{a}}(\theta)\left(\tilde{\boldsymbol{a}}^{\mathrm{H}}(\theta) \tilde{\boldsymbol{a}}(\theta)\right)^{-1} \tilde{\boldsymbol{a}}^{\mathrm{H}}(\theta)$ 是 $\tilde{\boldsymbol{a}}(\theta) \triangleq \hat{\boldsymbol{Q}}^{-\frac{\mathrm{H}}{2}} \boldsymbol{a}(\theta)$ 的投影矩阵, $\hat{\boldsymbol{Q}}^{-\frac{1}{2}}$ 和 $\hat{\boldsymbol{Q}}^{-\frac{\mathrm{H}}{2}}$ 分别是矩阵 $\hat{\boldsymbol{Q}}^{-1}$ 通过 Cholesky 分解得到的上三角矩阵和下三角矩阵.

观察式 (24) 知, 利用信道估计 $\hat{\boldsymbol{H}}$ 的任一列都可以得到目标角度估计 $\hat{\theta}$. 然而, 由于存在 RCS 起 伏, 信道衰落 $\boldsymbol{\alpha}$ 的随机变化将影响角度估计的性能. 特别地, 当 $\hat{\alpha}_{k} \rightarrow 0$ 时, 有 $\hat{\boldsymbol{h}}_{k} \rightarrow \mathbf{0}$, 此时, 目标回 波信号非常微弱, 可能无法得到可以接受的角度估计性能. 仿真实验表明, 使用 $\hat{\boldsymbol{H}}$ 的多个列可以克服 RCS 起伏, 并提高估计精度. 使用整个信道矩阵估计信息 $\hat{\boldsymbol{H}}$, 则目标角度估计为

$$
\hat{\theta}=\arg \max _{\theta} \mathcal{S}(\theta), \quad \mathcal{S}(\theta)=\operatorname{tr}\left\{\hat{\boldsymbol{H}}^{\mathrm{H}} \hat{\boldsymbol{Q}}^{-\frac{1}{2}} \boldsymbol{\Pi} \hat{\boldsymbol{Q}}^{-\frac{\mathrm{H}}{2}} \hat{\boldsymbol{H}}\right\} .
$$

\section{4 空域滤波解释}

考虑均匀白噪声 (空域白和时域白) 的情况, 即各接收阵元处的噪声功率相等, $\boldsymbol{Q}=\sigma_{v}^{2} \boldsymbol{I}, \sigma_{v}^{2}$ 是噪 声平均功率. 类似周期图法的滤波器组解释的推导 ${ }^{[24]}$, 在给定角度 $\tilde{\theta}$, 式 (24) 中的函数可以表示为

$$
\mathcal{S}_{k}(\tilde{\theta})=\frac{\left\|\boldsymbol{a}^{\mathrm{H}}(\tilde{\theta}) \hat{\boldsymbol{h}}_{k}\right\|^{2}}{\sigma_{v}^{2} \boldsymbol{a}^{\mathrm{H}}(\tilde{\theta}) \boldsymbol{a}(\tilde{\theta})}=\beta^{2}\left\|\boldsymbol{a}^{\mathrm{H}}(\tilde{\theta}) \boldsymbol{a}(\hat{\theta})\right\|^{2},
$$

其中, $\hat{\boldsymbol{h}}_{k}=\hat{\alpha}_{k} \boldsymbol{a}(\hat{\theta}), \beta=\frac{\left|\hat{\alpha}_{k}\right|}{\sqrt{M \sigma_{v}}}$. 这里用到了 $\boldsymbol{a}^{\mathrm{H}}(\tilde{\theta}) \boldsymbol{a}(\tilde{\theta})=M$. 式 (26) 可进一步改写为

$$
\mathcal{S}_{k}(\tilde{\theta})=\beta^{2}\left\|\sum_{m=0}^{M-1} \hat{h}_{k, m} e^{-j(M-1-m) \tilde{\phi}}\right\|^{2}=\left\|\sum_{m=0}^{M-1} \hat{h}_{k, m} f_{(M-1)-m}\right\|^{2},
$$


其中, $\hat{h}_{k, m}$ 是 $\boldsymbol{a}(\hat{\theta})$ 的第 $m$ 个元素, $\tilde{\phi} \triangleq-2 \pi d_{r} \sin (\tilde{\theta})$,

$$
f_{m}= \begin{cases}\beta e^{j m \tilde{\phi}}, & m=0, \ldots,(M-1), \\ 0, & \text { 其他. }\end{cases}
$$

于是可以将空域滤波器 $\left\{f_{m}\right\}$ 的传递函数表示为

$$
\mathcal{F}(\phi)=\sum_{m=0}^{M-1} f_{m} e^{-j m \phi}=\beta e^{j\left(\frac{M-1}{2}\right)(\tilde{\phi}-\phi)} \frac{\sin \left(\frac{M}{2}(\tilde{\phi}-\phi)\right)}{\sin \left(\frac{1}{2}(\tilde{\phi}-\phi)\right)}=\beta e^{j\left(\frac{M-1}{2}\right)(\tilde{\phi}-\phi)} W(\tilde{\phi}-\phi),
$$

其中, $W(\phi)=\frac{\sin \left(\frac{M}{2} \phi\right)}{\sin \left(\frac{1}{2} \phi\right)}$ 是方向图函数 ${ }^{[25]}$.

当时域数据长度趋于无穷时, 周期图的峰值位置是噪声中的正弦信号的一致估计 ${ }^{[24]}$. 相应地, 对 空域信号, 当 $N=1$, 而阵元数 $M \rightarrow \infty$ 时, 周期图法将得到角度的一致估计. 注意到在这种情况下, 从理论上讲, 非零的随机信道衰落 $\alpha_{k}$ 只影响函数 $\mathcal{S}(\theta)$ 的幅度, 而不会影响角度 $\theta$ 的估计. 由于可以 从空域滤波的角度解释上一节给出的渐近 ML 方法, 下文将式 (25) 称为谱方法.

\section{5 多项式求根法}

由于接收阵列是均匀线阵, 因此, 式 (24) 可进一步简化为多项式求根问题 ${ }^{[21-23]}$. 特别地, 利用定 义 $\phi \triangleq 2 \pi d_{r} \sin (\theta)$, 接收阵列导向向量可记为

$$
\boldsymbol{a} \triangleq \boldsymbol{a}(\phi)=\left[\begin{array}{llll}
1 & e^{-j \phi} & \ldots & e^{-j(M-1) \phi}
\end{array}\right]^{\mathrm{T}} .
$$

定义矩阵

$$
\boldsymbol{C}=\left[\begin{array}{cccc}
c & -c^{*} & & \\
& c & -c^{*} & \\
& & \ddots & \ddots \\
& & c & -c^{*}
\end{array}\right] \in \boldsymbol{C}^{(M-1) \times M},
$$

其中, $c$ 是与 $\theta$ 有关的复变量, $(\cdot)^{*}$ 表示复共轭. 当一阶多项式 $c-c^{*} z$ 的根 $z=e^{-j \phi}$ 时, 向量 $\boldsymbol{a}$ 在矩 阵 $\boldsymbol{C}$ 的零空间 (null Space) 中, 即 $\boldsymbol{C a}=\mathbf{0}, \mathbf{0}$ 表示零向量. 由于多项式的根在单位圆上, 因此多项式 系数可以表示为 $c=\kappa e^{-j \phi / 2}$ 和 $c^{*}=\kappa e^{j \phi / 2}, \kappa$ 是任意尺度常数, $\kappa$ 不影响等式 $\boldsymbol{C a}=\mathbf{0}$. 不失一般性, 调整尺度 $\kappa$ 使得 $c$ 的实部为 1 , 即 $c=1+j c_{i}$. 于是, 矩阵 $C$ 只与虚部 $c_{i}$ 有关.

观察到矩阵乘积 $\boldsymbol{C} \hat{\boldsymbol{Q}}^{1 / 2}$ 的秩为 $M-1$, 并且 $\left(\hat{\boldsymbol{Q}}^{1 / 2} \boldsymbol{C}^{\mathrm{H}}\right)^{\mathrm{H}} \hat{\boldsymbol{Q}}^{-\mathrm{H} / 2} \boldsymbol{a}=\mathbf{0}$. 因此, $\hat{\boldsymbol{Q}}^{1 / 2} \boldsymbol{C}^{\mathrm{H}}$ 的列向量张 成 $\tilde{\boldsymbol{a}}=\hat{\boldsymbol{Q}}^{-\frac{\mathrm{H}}{2}} \boldsymbol{a}(\theta)$ 的零空间. 于是, $\tilde{\boldsymbol{a}}$ 的正交补投影矩阵 $\boldsymbol{\Pi}^{\perp}$ 可以表示为

$$
\boldsymbol{\Pi}^{\perp} \triangleq \boldsymbol{I}-\boldsymbol{\Pi}=\hat{\boldsymbol{Q}}^{1 / 2} \boldsymbol{C}^{\mathrm{H}}\left(\boldsymbol{C} \hat{\boldsymbol{Q}} \boldsymbol{C}^{\mathrm{H}}\right)^{-1} \boldsymbol{C} \hat{\boldsymbol{Q}}^{\mathrm{H} / 2},
$$

因此, 角度 $\theta$ 的估计可以重写为

$$
\hat{\theta}=\arg \min _{\theta} \hat{\boldsymbol{h}}_{k}^{\mathrm{H}} \hat{\boldsymbol{Q}}^{-\frac{1}{2}} \boldsymbol{\Pi}^{\perp} \hat{\boldsymbol{Q}}^{-\frac{\mathrm{H}}{2}} \hat{\boldsymbol{h}}_{k}=\arg \min _{\theta} \hat{\boldsymbol{h}}_{k}^{\mathrm{H}} \boldsymbol{C}^{\mathrm{H}}\left(\boldsymbol{C} \hat{\boldsymbol{Q}} \boldsymbol{C}^{\mathrm{H}}\right)^{-1} \boldsymbol{C} \hat{\boldsymbol{h}}_{k},
$$

其中, 可以用相应的根 $-N(\operatorname{root}-N)$ 一致估计代替矩阵 $\boldsymbol{C}$ 而不影响估计值的大样本性能 ${ }^{[26]}$. 因为 $\boldsymbol{C h}_{k}=\mathbf{0}$, 且 $\boldsymbol{h}_{k}-\hat{\boldsymbol{h}}=O(1 / \sqrt{N})$, 有

$$
\boldsymbol{C} \hat{\boldsymbol{h}}_{k}=O(1 / \sqrt{N})
$$


其中, $O(1 / \sqrt{N})$ 表示随机变量在根均方 (root-mean-square) 意义下的 (渐近) 阶数 ${ }^{[22]}$. 因此, 式 (33) 简化为二次函数的极小化问题

$$
\hat{c}_{i}=\arg \min _{c_{i}} \hat{\boldsymbol{h}}_{k}^{\mathrm{H}} \boldsymbol{C}^{\mathrm{H}} \boldsymbol{C} \hat{\boldsymbol{h}}_{k},
$$

其中, $c_{i}$ 是多项式系数估计值 $\hat{c}$ 的虚部. 于是, 角度 $\theta$ 的估计为

$$
\hat{\theta}=\arcsin \left(\frac{-\angle \hat{c}^{2}}{2 \pi d_{r}}\right)
$$

其中, $\arcsin (\cdot)$ 和 $\angle(\cdot)$ 分别表示反正弦函数和复数的相角 (在主值区间中). 与谱方法 (25) 类似, 利用 $\hat{\boldsymbol{H}}$ 的所有列, $c_{i}$ 的估计为

$$
\hat{c}_{i}=\arg \min _{c_{i}} \operatorname{tr}\left\{\hat{\boldsymbol{H}}^{\mathrm{H}} \boldsymbol{C}^{\mathrm{H}} \boldsymbol{C} \hat{\boldsymbol{H}}\right\} .
$$

式 (35) 和 (37) 都可以高效地数值求解. 与式 (35) 相比, 式 (37) 通常具有更好的估计性能. 将角度估 计 $\hat{\theta}$ 代入式 $(23)$, 即可得到衰落向量的估计.

\section{6 仿真结果}

本节将通过仿真实验研究算法的性能. 使用 $M=20$ 阵元的均匀线阵作为接收阵列, 阵元间距为 二分之一波长. 目标位于 $\theta=0^{\circ}$. 定义衰落向量估计的均方误差 (mean square error, MSE) 为

$$
\operatorname{MSE}(\hat{\boldsymbol{\alpha}}) \triangleq \frac{1}{T} \sum_{t=1}^{T}\left(\frac{\left\|\hat{\boldsymbol{\alpha}}_{t}-\boldsymbol{\alpha}_{t}\right\|}{\left\|\boldsymbol{\alpha}_{t}\right\|}\right)^{2},
$$

其中, $\boldsymbol{\alpha}_{t}$ 和 $\hat{\boldsymbol{\alpha}}_{t}$ 分别是第 $t$ 次实验中真实的和估计的衰落向量, $T=500$ 是独立实验次数. 类似地, 定 义角度估计的 MSE 为

$$
\operatorname{MSE}(\hat{\theta}) \triangleq \frac{1}{T} \sum_{t=1}^{T}\left\|\hat{\theta}_{t}-\theta_{t}\right\|^{2} .
$$

在仿真实验中, 采用与文献 [9] 相同的正交发射信号, 发射信号向量 $\boldsymbol{s}[n]$ 的各分量是零均值的, 且协方 差矩阵为 $\boldsymbol{R}_{\boldsymbol{s} s}=E\left\{\boldsymbol{s}[n] \boldsymbol{s}^{\mathrm{H}}[n]\right\}=\sigma_{s}^{2} \boldsymbol{I}_{K}, \sigma_{s}^{2}$ 是发射信号的平均功率.

在第一个仿真实例中, 假设加性噪声向量 $\boldsymbol{v}[n]$ 是空间白和时间白的, 且协方差矩阵为 $\boldsymbol{Q}=\sigma_{v}^{2} \boldsymbol{I}_{M}$. 定义接收信噪比 ${ }^{[2]}$ 为 $\mathrm{SNR}=\frac{E\left\{\sigma_{s}^{2} K^{-1}\|\boldsymbol{\alpha}\|^{2}\right\}}{\sigma_{v}^{2}}=\frac{\sigma_{s}^{2}}{\sigma_{v}^{2}}$. 在每次独立实验中, 随机生成衰落向量 $\boldsymbol{\alpha}$, 并且在 $N$ 个快拍过程中保持不变. 对于估计器 (9) 和 (25), 首先按 $1^{\circ}$ 的间隔进行搜索, 并且在检测到的极值 点附近递归地优化角度估计值, 最终的搜索间隔为 $\left(10^{-5}\right)^{\circ}$. 在完成角度估计后, 利用式 (23) 实现衰落 向量的估计.

图 3 和 5 分别给出了在均匀噪声环境中, 不同 SNR 条件下, 角度估计和衰落向量估计的 MSE, 使用的快拍数为 $N=100$. 图 4 和 6 分别给出了 $\mathrm{SNR}=10 \mathrm{~dB}$ 时, 不同快拍数条件下的角度估计和衰 落向量估计的 MSE. 发射天线数目分别为 4 和 16(其中, 图 5 给出了 8 发射天线的情况). 图 5 和 6 给 出的仿真结果表明, 三种方法 (9), (25) 和 (37) 得到的角度估计性能都将随着信噪比和快拍数的增加 而改善; 谱方法 (25) 与延迟求和波束形成器 (9) 的性能相近, 而多项式求根法 (37) 性能略差. 图 3 和 4 则表明, 谱方法和多项式求根法对衰落向量的估计性能影响并不明显. 如果从计算效率考虑, 多项式 求根法是一种不错的渐近 ML 方法. 除此之外, 仿真结果还表明, 三种方法的估计性能会随着发射天 线数目的增加而有所改善. 


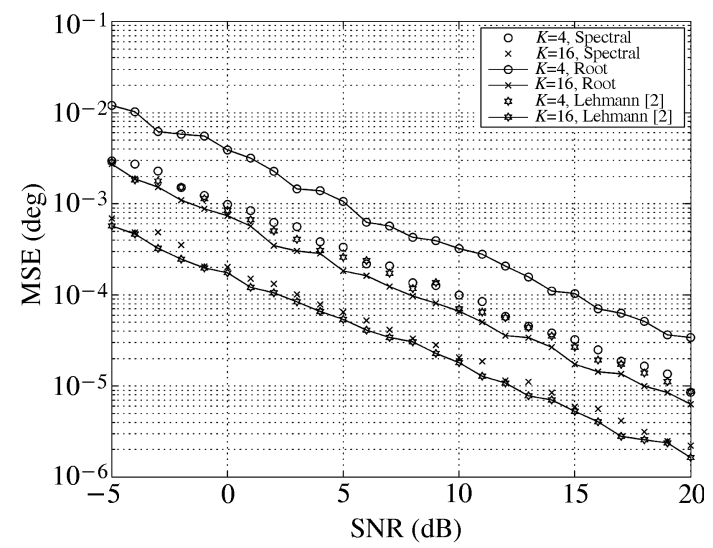

图 3 不同 SNR 情况下角度估计的 MSE, 快拍数 $N=100$, 发射天线数 $K=4$ 和 16

Figure 3 MSE of angle estimate with different SNRs. The number of snapshots is 100 . The numbers of transmit antennas are $K=4$ and 16

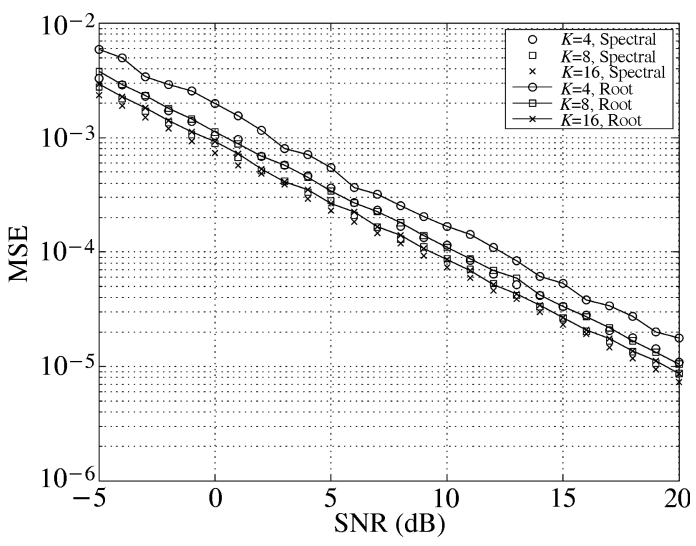

图 5 不同 SNR 情况下衰落向量估计的 MSE, 快拍 数 $N=100$, 发射天线数 $K=4,8$ 和 16

Figure 5 MSE of fading vector estimate with different SNRs. The number of snapshots is 100 .

The of numbers transmit antennas are $K=4$, 8 and 16

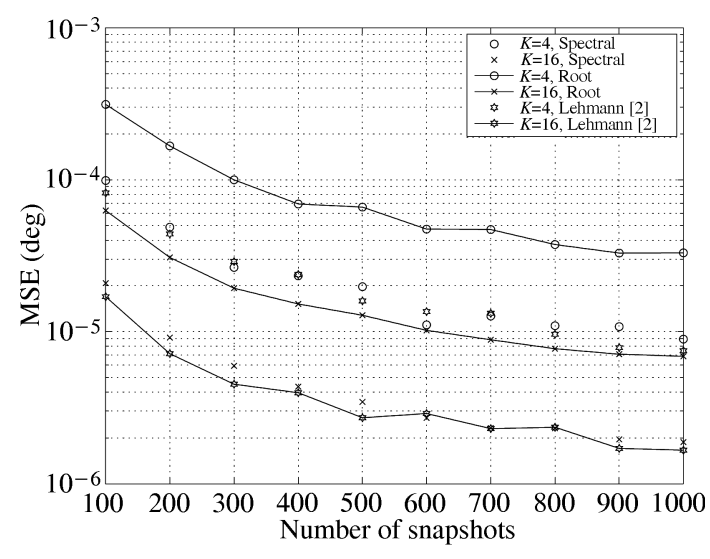

图 4 不同快拍数情况下角度估计的 MSE, 发射 天线数 $K=4$ 和 $16, \mathrm{SNR}=10 \mathrm{~dB}$

Figure 4 MSE of angle estimate with different numbers of snapshots. The numbers of transmit antennas are $K=4$ and $16, \mathrm{SNR}=10 \mathrm{~dB}$

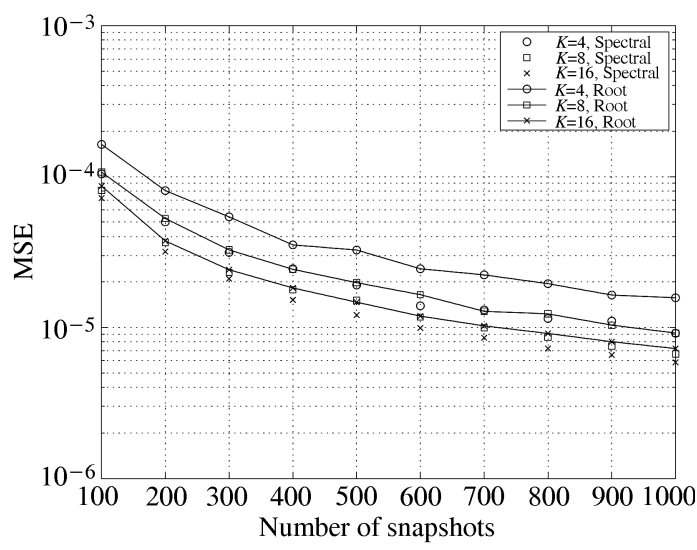

图 6 不同快拍数情况下衰落向量估计的 MSE, 发射天线数 $K=4$ 和 $16, \mathrm{SNR}=10 \mathrm{~dB}$.

Figure 6 MSE of fading vector estimate with different numbers of snapshots. The numbers of transmit antennas are $K=4$ and 16 , SNR

$$
=10 \mathrm{~dB}
$$

在第二个仿真实例中, 考虑非均匀噪声的情况, 即假设加性噪声向量 $\boldsymbol{v}[n]$ 是空间白和时间白的, 但是各接收阵元处的噪声功率不相等. 噪声协方差矩阵为对角阵 $\boldsymbol{Q}=\sigma_{v}^{2} \boldsymbol{D}$, 其中 $\boldsymbol{D}$ 是对角阵. 定义最 坏噪声功率比 (worst noise power ratio, WNPR) 为 ${ }^{[27]} \mathrm{WNPR} \triangleq \frac{\sigma_{\max }^{2}}{\sigma_{\min }^{2}}$, 其中, $\sigma_{\max }^{2}$ 和 $\sigma_{\min }^{2}$ 分别是最大 和最小的噪声功率. 这里, 选择主对角元素是在区间 $(0,10]$ 上均匀分布的随机变量, 使得 $\mathrm{WNPR}=20$. 在此实例中, 定义信噪比 $\mathrm{SNR}=\frac{\sigma_{s}^{2}}{\sigma_{v}^{2}}$. 注意, 算法没有利用噪声协方差矩阵的特殊结构. 


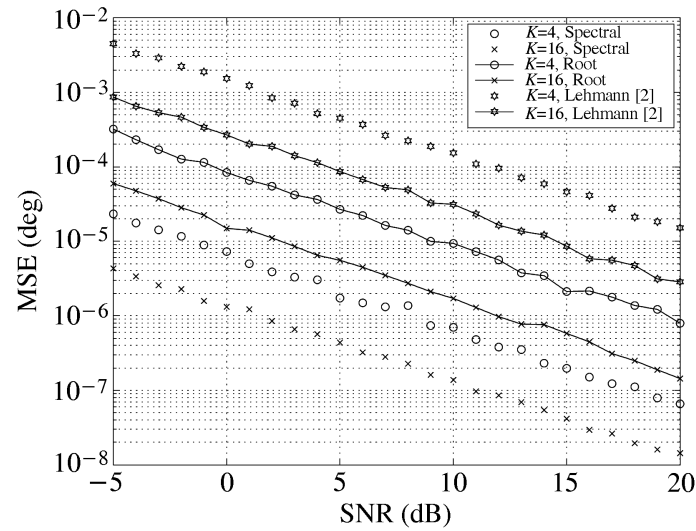

图 7 非均匀噪声环境中, 不同 SNR 情况下 的 MSE. 角度估计快拍数 $N=100$. 发射天线

$$
\text { 数 } K=4 \text { 和 } 16
$$

Figure 7 MSE of angle estimate with different SNRs in nonuniform noise environment. The number of snapshots is 100 . The numbers of transmit antennas are $K=4$ and 16

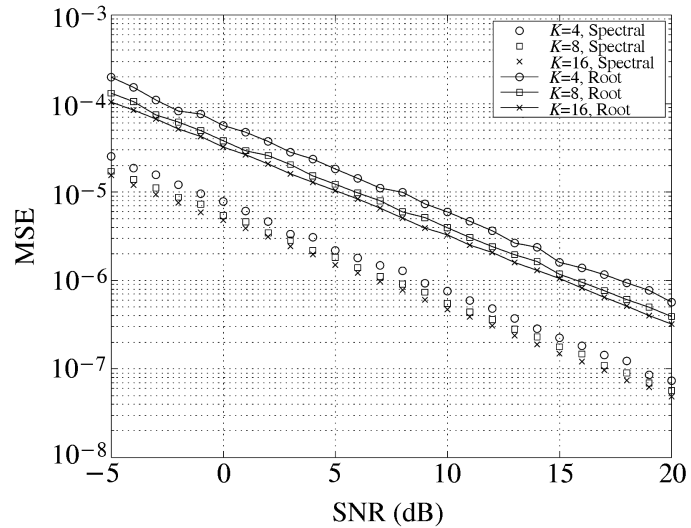

图 8 非均匀噪声环境中, 不同 SNR 情况下衰落 向量估计的 MSE. 快拍数 $N=100$. 发射天线数

$$
K=4,8 \text { 和 } 16
$$

Figure 8 MSE of fading vector estimate with different SNRs in nonuniform noise environment. The number of snapshots is 100 . The numbers of transmit antennas are $K=4,8$ and 16

图 7 和 8 分别给出了在非均匀噪声环境中, 不同 SNR 条件下, 角度估计和衰落向量估计的 MSE, 发射信号样本数为 $N=100$. 发射天线数目仍然分别为 4 和 16 (其中, 图 8 给出了 8 发射天线的情 况). 与第一个仿真实例的结果类似, 三种方法得到的角度估计性能都将随着信噪比和快拍数的增加而 改善; 谱方法的性能略优于多项式求根法, 但计算量较大. 值得注意的是, 在这种情况下, 谱方法和多 项式求根法的性能优于延迟求和波束形成器的性能.

\section{7 结束语}

本文讨论了发射空域分集, 相干接收的 MIMO 雷达模型及其最大似然参数估计问题. 本文推导了 两种渐近最大似然方法, 谱方法和多项式求根法, 并从空域滤波的角度解释了谱方法. 仿真实验验证 了两种算法的有效性, 其中, 谱方法的估计性能比多项式求根法性能略好, 但计算量较大. 仿真实验结 果也表明, 在非均匀噪声条件下, 本文推导的两种渐近方法的性能优于延迟求和波束形成器的性能.

\section{参考文献}

1 Fishler E, Haimovich A, Blum R S, et al. Spatial diversity in radars-models and detection performance. IEEE Trans Signal Process, 2006, 54: 823-838

2 Lehmann N H, Fishler E, Haimovich A M, et al. Evaluation of transmit diversity in MIMO-radar direction finding. IEEE Trans Signal Process, 2007, 55: 2215-2225

3 Haimovich A M, Blum R S, Cimini L J. MIMO radar with widely separated antennas. IEEE Signal Process Mag, 2008, 25: $116-129$ 
4 Bekkerman I, Tabrikian J. Target detection and localization using MIMO radars and sonars. IEEE Trans Signal Process, 2006, 54: 3873-3883

5 Xu L, Li J, Stoica P. Adaptive techniques for MIMO radar. In: 4th IEEE Workshop Sensor Array Multi-Channel Processing. Waltham, MA: IEEE Press, 2006. 258-262

6 Xu L, Li J. Iterative generalized-likelihood ratio test for MIMO radar. IEEE Trans Signal Process, 2007, 55: 2375-2385

7 Li J, Stoica P, Xu L, et al. On parameter identifiability of MIMO radar. IEEE Signal Process Lett, 2007, 14: 968-971

8 Fuhrmann D R, Antonio G S. Transmit beamforming for MIMO radar systems using partial signal correlation. In: Conference Record of the 38th Asilomar Conference on Signals, Systems and Computers. New York: IEEE Press, 2004. 295-299

9 Stoica P, Li J, Xie Y. On probing signal design for MIMO radar. IEEE Trans Signal Process, 2007, 55: 4151-4161

10 Li J, Xu L, Stoica P, et al. Range compression and waveform optimization for MIMO radar: a Cramer-Rao bound based study. IEEE Trans Signal Process, 2008, 56: 218-232

11 Yang Y, Blum R S. MIMO radar waveform design based on mutual information and minimum mean-square error estimation. IEEE Trans Aerosp Electro Syst, 2007, 43: 330-343

12 Li J, Stoica P. MIMO radar with colocated antennas. IEEE Signal Process Mag, 2007, 24:106-114

13 He Z S, Han C L, Liu B. MIMO radar and its technical characteristic analyses. Acta Electron Sin, 2005, 33: 143-147

14 Chen C Y, Vaidyanathan P P. MIMO radar space-time adaptive processing using prolate spheroidal wave functions. IEEE Trans Signal Process, 2008, 56: 623-635

15 He Q, Blum R S, Haimovich A M. Non-coherent MIMO radar for location and velocity estimation: more antennas means better performance. IEEE Trans Signal Process, 2010, 58: 3661-3680

16 He Q, Blum R S, Godrich H, et al. Target velocity estimation and antenna placement for MIMO radar with widely separated antennas. IEEE J Sel Topic Signal Process, 2010, 4: 79-100

17 He Q, Blum R S. Cramer-Rao bound for MIMO radar target localization with phase errors. IEEE Signal Processing Lett, 2010, 17: 83-86

18 Tang J, Wu Y, Peng Y N, et al. On detection performance and system configuration of MIMO radar. Sci China Inf Sci, 2009,52: 1250-1257

19 Tang J, Wu Y, Peng Y N, et al. On detection performance of MIMO radar for Rician target. Sci China Inf Sci, 2009, 52: $1456-1465$

20 Guan J, Huang Y. Detection performance analysis for MIMO radar with distributed apertures in Gaussian colored noise. Sci China Inf Sci, 2009, 52: 1688-1696

21 Xia W. Research on Models and Signal Processing for MIMO Radars. Dissertation for the Doctoral Degree. Chengdu: University of Electronic Science and Technology of China, 2008

22 Li J, Halder B, Stoica P, et al. Computationally efficient angle estimation for signals with known waveforms. IEEE Trans Signal Process, 1995, 43: 2154-2163

23 Swindlehurst A L, Stoica P. Maximum likelihood methods in radar array signal processing. Proc IEEE, 1998, 86: $421-441$

24 Stoica P, Moses R. Introduction to Spectral Analysis. Upper Saddle River, NJ: Prentice-Hall, 1997

25 Simon Haykin, ed. Array Signal Processing. Englewood Cliffs, NJ: Prentice-Hall, 1985

26 Stoica P, Sharman K C. Maximum likelihood methods for direction-of-arrival estimation. IEEE Trans Acoust Speech Signal Process, 1990, 38: 1132-1143

27 Pesavento M, Gershman A B. Maximum-likelihood direction-of-arrival estimation in the presence of unknown nonuniform noise. IEEE Trans Signal Process, 2001, 49: 1310-1324 


\title{
On the maximum likelihood method for target localization using MIMO radars
}

\author{
XIA Wei*, HE ZiShu \& LIAO YuYu \\ School of Electronic Engineering, University of Electronic Science and Technology of China, Chengdu 611731, \\ China \\ *E-mail: wx@uestc.edu.cn
}

\begin{abstract}
A multiple-input multiple-output (MIMO) radar uses multiple antennas to simultaneously transmit multiple independent probing signals, and uses multiple antennas to receive the backscattered signals. The modeling of MIMO radar with transmit spatial diversity and coherent reception is addressed herein. The maximum likelihood (ML) method for parameter estimation using MIMO radars is considered, and two approximate ML algorithms are proposed. In the uniform noise scenario, one of the proposed algorithms performs similarly to the delay-and-sum beamformer which is optimal in the ML sense in single target case, while it outperforms the other proposed approximate ML algorithm at the cost of more computational load. In the non-uniform noise scenario, the proposed approximate ML algorithms both outperform the delay-and-sum beamformer. The efficiency of the proposed methods is validated by the simulation results.
\end{abstract}

Keywords maximum-likelihood, multiple-input multiple-output (MIMO) radars, parameter estimation 